\title{
The ATLAS Trigger and Data Acquisition Upgrades for the High-Luminosity LHC (HL-LHC)
}

\author{
M. Valente, on behalf of the ATLAS collaboration \\ Département de Physique Nucléaire et Corpusculaire (DPNC) \\ Université de Genève \\ E-mail: marco.valente@cern.ch
}

The ATLAS experiment at CERN has started the construction of upgrades for the HighLuminosity LHC (HL-LHC), with collisions due to start in 2026. In order to deliver an order of magnitude more data than previous LHC runs, $7 \mathrm{TeV}$ protons will collide at $14 \mathrm{TeV}$ with an instantaneous luminosity of up to $7.5 \times 10^{34} \mathrm{~cm}^{-2} \mathrm{~s}^{-1}$, resulting in much higher pileup and data rates than the current experiment was designed to handle. While this is essential to realise the physics program, it presents a huge challenge for the detector, trigger, data acquisition and computing. The detector upgrades themselves present new requirements and opportunities for the trigger and data acquisition system. The approved baseline design of the TDAQ upgrade comprises: a hardware-based low-latency real-time trigger operating at $40 \mathrm{MHz}$, data acquisition which combines custom readout with commodity hardware and networking to deal with $5.2 \mathrm{~TB} / \mathrm{s}$ input, and an event filter running at $1 \mathrm{MHz}$, which combines offline-like algorithms on a large set of commodity servers and hardware tracking. Commodity servers and networks are used, with custom ATCA boards, high speed links and powerful FPGAs deployed in the low-latency parts of the system. Offline-style clustering and jet-finding in FPGAs, and track reconstruction with Associative Memory ASICs and FPGAs are designed to combat pileup in the hardware trigger and the event filter respectively. This document reports recent progress on the design of the system and the performance on key physics processes.

European Physical Society Conference on High Energy Physics - EPS-HEP2019 -

10-17 July, 2019

Ghent, Belgium 


\section{Introduction}

The High-Luminosity LHC (HL-LHC) is an upgrade of the Large Hadron Collider (LHC) aiming at increasing the instantaneous luminosity of the LHC by at least a factor of five with respect to its design value and it is planned to be operative starting from 2026. For this to be achieved, the number of simultaneous p-p collisions (pileup) will be increased to 200 average interactions per bunch-crossing and the ATLAS experiment [1] is expected to collect a total integrated luminosity of $3000 \mathrm{fb}^{-1}$ after ten years of operation, improving sensitivity to extremely rare new physics channels.

In order to meet these challenging requirements and to fully exploit the physics potential of the HL-LHC, the ATLAS Trigger and Data Acquisition system (TDAQ) will need to be upgraded, with the aim to maintain during the HL-LHC program trigger selection requirements as close as possible to the ones currently employed in Run 2 and 3 [3]. This document aims at reviewing the major upgrades of the HL-LHC ATLAS TDAQ system.

\section{The Phase-II ATLAS TDAQ upgrade}

The Phase-II upgrade of the ATLAS TDAQ system is complementary to the different upgrades of the ATLAS subdetectors, in particular:

- The new Inner Tracker (ITk) that will make the ATLAS tracking system fully based on silicon devices with an extended pseudorapidity acceptance up to $\eta=4.0$.

- The upgraded calorimeter readout electronics of both Liquid Argon (LAr) and Tile calorimeters in order to meet the Phase-II requirements in terms of trigger rates, latency and to feed the trigger with full-granularity information.

- The upgrade of the Muon Trigger Chambers and readout electronics in order to meet the Level-0 trigger requirements and to extend the solid angle coverage.

In addition to these features, the ATLAS HL-LHC TDAQ upgrade will also rely largely on the TDAQ upgrades made during the Long Shutdown 2 such as the upgraded calorimeter trigger processors and Feature Extractors (FEX) [2].

Two possible architectures have been defined for the Phase-II upgrade of the ATLAS TDAQ system [3]:

- A baseline architecture composed of a hardware-based Level-0 (L0) trigger running at $40 \mathrm{MHz}$ input rate and a CPU farm-based Event Filter (EF) assisted by hardware-based track reconstruction and running at $1 \mathrm{MHz}$ input rate.

- An evolved version of the baseline architecture where an intermediate Level-1 (L1) trigger with 2-4 MHz input rate provides an additional filtering step between the Level-0 and Event Filter. In this scenario, the EF input rate is reduced to $800-600 \mathrm{kHz}$ compared to the baseline scenario.

Both architectures are designed to provide a $10 \mathrm{kHz}$ output rate. A simplified schematic of the baseline architecture design is reported in Figure 1a. 


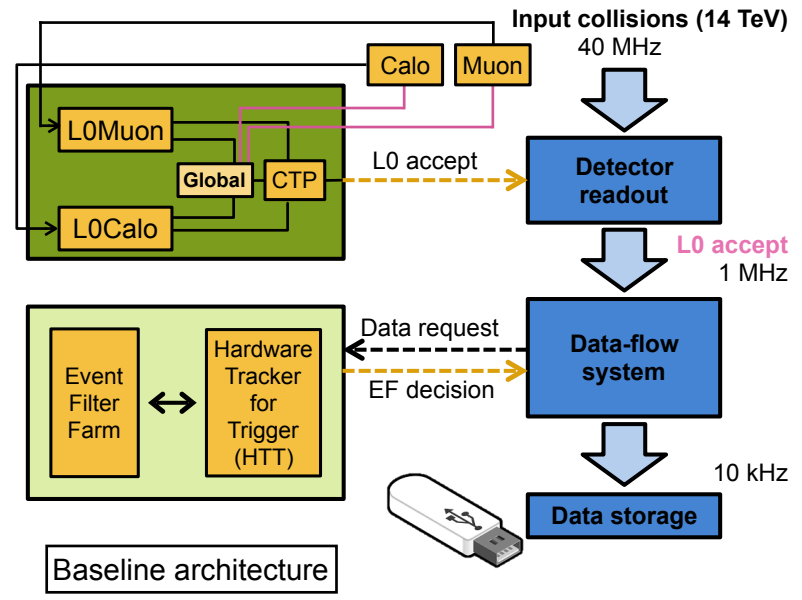

(a) Baseline design Phase-II ATLAS TDAQ architecture.

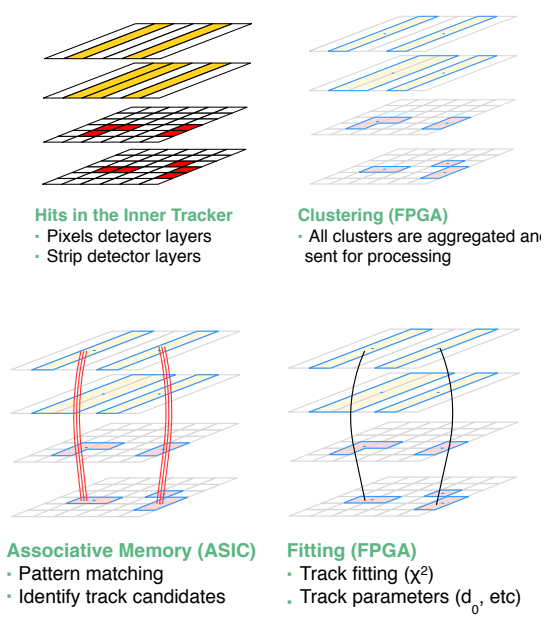

(b) Associative-memory-based HTT tracking.

Figure 1: Schematic of baseline design of the Phase-II ATLAS TDAQ architecture and associativememory-based tracking sequence performed by the Hardware Track Trigger (HTT). [4].

\subsection{Level-0 trigger}

The Level-0 trigger represents the first step of the new ATLAS trigger system and it will reduce the $40 \mathrm{MHz}$ HL-LHC rate to the $1 \mathrm{MHz}$ output in the baseline trigger design. This trigger is based on a hardware system composed of the Level-O Calorimeter Trigger (L0Calo), the Level-0 Muon Trigger (LOMuon), the Global Trigger and the Central Trigger (CTP) sub-systems [3].

LOCalo provides reconstruction of calorimeter objects using different FPGA-based FEXs. These systems allow to create complex and high-granularity objects exploiting the full calorimeter coverage of ATLAS through LAr calorimeter super-cells ${ }^{1}$ and Tile $\eta \times \phi=0.1 \times 0.1$ trigger towers. LOMuon provides muon reconstruction with sophisticated algorithms and extended $\eta$ coverage thanks to the entirely new muon trigger electronics and readout chain. Moreover, the L0Muon upgrade also includes the first use of precision Muon Detectors (MDTs) in the hardware-level trigger.

New functionality of the upgraded Level-0 trigger is offered by high-rate processing at the Global Trigger. After L0Calo, this system improves calorimeter objects using full-granularity energy data coming directly from upgraded calorimeter pre-processors and implementing offline-like algorithms such as topological clustering, anti- $k_{t}$-based jet-finding, refined electron and photon identification, tau lepton identification and calorimeter-based pileup suppression [3]. Additionally, the Global Trigger uses the inputs from the L0Calo and L0Muon systems in order to apply topological selections based on $p_{\mathrm{T}}$ and angular requirements, a feature offered today by the ATLAS L1Topo system.

\subsection{Event Filter and Hardware Track Trigger (HTT)}

The Event Filter system consists of a CPU-based processing farm and a Hardware Track Trigger (HTT) co-processor. The main function of the EF is to refine the selection of the $1 \mathrm{MHz}$ L0

\footnotetext{
${ }^{1}$ These are LAr calorimeter regions formed by summing $E_{\mathrm{T}}$ from cells that are adjacent in $\eta$ and $\phi$.
} 


\begin{tabular}{l|c|c|c|c}
\hline Trigger name & Physics signatures & $\begin{array}{c}\text { Threshold } \\
{[\text { Run 1] }(\mathrm{GeV})}\end{array}$ & $\begin{array}{c}\text { Threshold } \\
{[\text { Run 2] }(\mathrm{GeV})}\end{array}$ & $\begin{array}{c}\text { Threshold } \\
{[\mathrm{HL}-\mathrm{LHC}](\mathrm{GeV})}\end{array}$ \\
\hline Isolated single e & $W H, Z H, t \bar{t}$, & 25 & 27 & 22 \\
Isolated single $\mu$ & EW SUSY & 25 & 27 & 20 \\
Di- $\gamma$ & $H \rightarrow \gamma \gamma, H H \rightarrow b \bar{b} \gamma \gamma$ & 25,25 & 25,25 & 25,25 \\
Di- $\tau$ & $H \rightarrow \tau \bar{\tau}$, EW SUSY & 40,30 & 40,30 & 40,30 \\
Four-jet w/ b-jets & $H H \rightarrow b \bar{b} b \bar{b}$, RPV SUSY & 45 & 45 & 65 \\
$H_{\mathrm{T}}$ & SUSY & 700 & 700 & 375 \\
MET & $Z H \rightarrow v \bar{v} b \bar{b}$, Dark Matter & 150 & 200 & 200 \\
\hline
\end{tabular}

Table 1: Key trigger thresholds and physics signatures for $1 \mathrm{MHz}$ Level-0 trigger rate [3].

output events using sophisticated offline-like reconstruction techniques allowing to reduce the final output rate to a proper disk storage value. The HTT includes regional (rHTT) and global (gHTT) track reconstruction, where regional tracking is made in specific Regions of Interest (RoIs) limited to approximately $10 \%$ of the total Inner Tracker data while global tracking exploits the full tracker coverage, but only for $10 \%$ of L0 selected events. Since rHTT can run at higher frequencies than gHTT, the selections are applied in sequence in order to reject events as early as possible during their processing. However, both regional and global tracking rely on associative memory techniques, where tracking is accelerated using pre-loaded pattern banks to be matched to the tracker output (see Figure 1b). The final output of the EF is fixed at $10 \mathrm{kHz}$.

The EF trigger decision is finally communicated to the Dataflow system, which transfers accepted events to permanent storage.

\section{Trigger thresholds and impact on key physics items}

The increased luminosity provided by the HL-LHC aims at exploring very rare physics channels providing precise measurements of the Higgs sector, searches for new particles with low interaction rates and precise measurements of electro-weak (EW) processes. In this context, the Phase-II upgrade of the ATLAS TDAQ system will be essential to maintain the current EW-scale physics thresholds and to select efficiently physics signals.

Table 1 reports some key trigger thresholds and physics signatures of the ATLAS HL-LHC physics program. This table shows the ability of the upgraded TDAQ system to ensure trigger thresholds similar to or lower than the current Run 2 values, allowing to fully exploit the increased luminosity of the HL-LHC. In this context, Figure 2a clearly illustrates how the novel L0 Global system will reduce the thresholds of four-jet triggers, allowing to select more signal events for key physics items such as $H H \rightarrow b \bar{b} b \bar{b}$. Additionally, Figure $2 \mathrm{~b}$ shows the large impact of the HTT upgrade on $E_{\mathrm{T}}^{\text {miss }}$ triggers, an essential signature for $Z H \rightarrow v \bar{v} b \bar{b}$, SUSY and Dark Matter searches. These improvements are possible due to the pileup rejection capabilities offered by regional and global tracking. HTT will also play a major role in the reduction of pileup effects and trigger rates of key hadronic signatures such as $H H \rightarrow b \bar{b} b \bar{b}$ and electro-weak SUSY. 


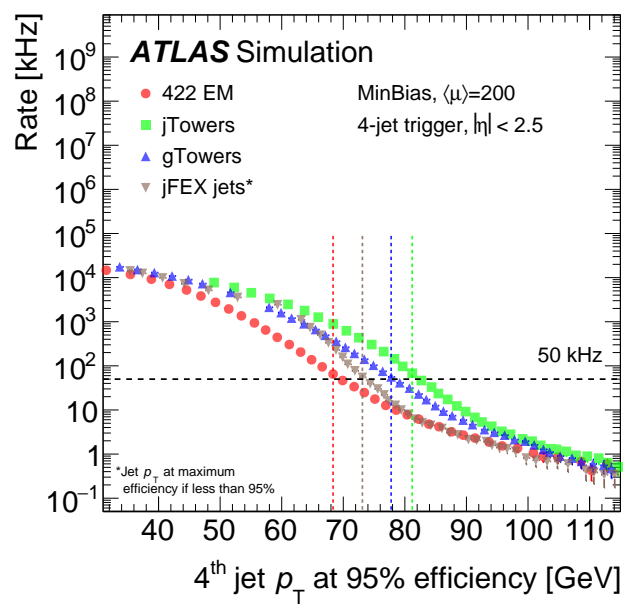

(a) L0 trigger rate versus threshold at $95 \%$ efficiency for three-jet and four-jet triggers.

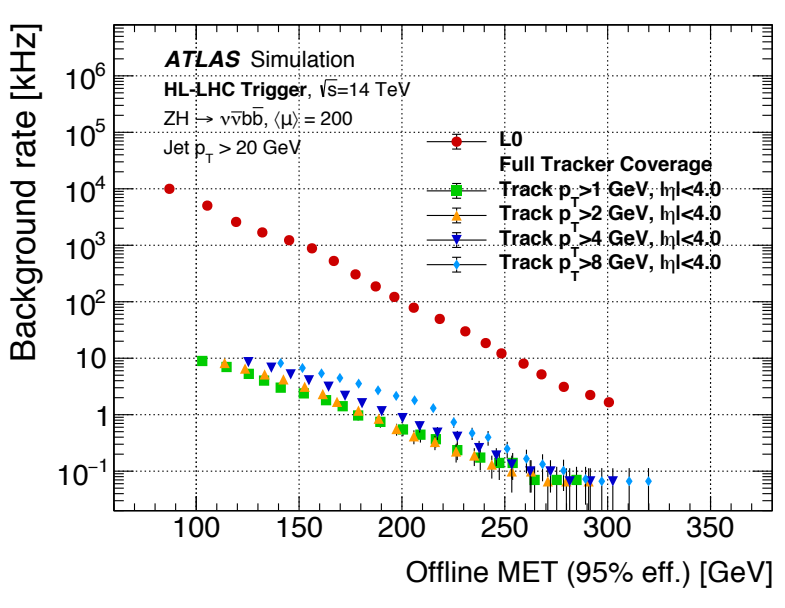

(b) Rate vs offline $E_{\mathrm{T}}^{\text {miss }}$ cut at $95 \%$ selection efficiency of $Z H \rightarrow v \bar{v} b \bar{b}$ signal for different track $p_{\mathrm{T}}$ requirements.

Figure 2: Impact of L0 global topoclustering and different HTT tracking requirements on four-jet and $E_{\mathrm{T}}^{\text {miss }}$ triggers [3].

\section{Conclusions}

The HL-LHC will significantly increase the physics potential of the ATLAS experiment thanks to the unprecedented data rates it will provide. In order to fully exploit this enhanced capability, major upgrades of the ATLAS trigger system are necessary. In this context, the current plans for the TDAQ Phase-II upgrade will provide the ATLAS trigger with the required bandwidth and processing capabilities for efficiently selecting events at high luminosity. This upgrade represents an important ingredient for the success of the ATLAS HL-LHC physics program and work has started towards the construction of the first system prototypes.

\section{References}

[1] ATLAS Collaboration. The ATLAS Experiment at the CERN Large Hadron Collider. JINST, 3:S08003, 2008.

[2] ATLAS Collaboration. Technical Design Report for the Phase-I Upgrade of the ATLAS TDAQ System. Sep 2013. CERN-LHCC-2013-018, ATLAS-TDR-023.

https://cds.cern.ch/record/1602235.

[3] ATLAS Collaboration. Technical Design Report for the Phase-II Upgrade of the ATLAS TDAQ System. Sep 2017. CERN-LHCC-2017-020, ATLAS-TDR-029.

https://cds.cern.ch/record/2285584.

[4] R. Poggi. Design of the ATLAS Phase-II hardware-based tracking processor. Nuclear Instruments and Methods in Physics Research Section A: Accelerators, Spectrometers, Detectors and Associated Equipment, 936:305 - 307, 2019. Frontier Detectors for Frontier Physics: 14th Pisa Meeting on Advanced Detectors. 